\title{
Safety of Janus Kinase Inhibitors in Older Patients: A Focus on the Thromboembolic Risk
}

\author{
Suraj Rajasimhan ${ }^{1} \cdot$ Omer Pamuk ${ }^{2} \cdot$ James D. Katz ${ }^{2}$ (D)
}

Published online: 8 June 2020

(c) The Author(s) 2020

\begin{abstract}
The Janus kinase (JAK)-signal transducer and activator of transcription (STAT) pathway is a membrane-to-nucleus signaling cascade that effects activation of gene transcription. JAK inhibitors have demonstrated effectiveness in autoimmune diseases such as rheumatoid arthritis. An increased risk of infection, mainly varicella-zoster reactivation, with these new agents is of concern. Comorbid conditions, along with pharmacokinetic variations in drug metabolism in the older population, further increase the risk of adverse outcomes. Newly raised concerns for potential adverse effects such as deep vein thrombosis and pulmonary embolism are essential considerations for clinicians. Older patients are at increased risk because of multiple comorbid conditions and pharmacokinetic changes related to drug metabolism and excretion. Both the US FDA and the European Medicines Agency have issued warnings regarding this risk. These warnings highlight individuals aged $>50$ years with concomitant cardiovascular risk factors. Furthermore, the FDA released a black box warning for increased thromboembolic risk associated with JAK inhibitors. As the use of these drugs increases, a solid understanding of adverse effects and risks is critical to those treating older adults.
\end{abstract}

\section{Key Points}

Thromboembolic risk is an important and emerging consideration for clinicians who prescribe Janus kinase (JAK) inhibitors. Older patients with rheumatoid arthritis are at increased thromboembolic risk because of age and comorbid conditions.

The warnings issued by the US FDA and the European Medicines Agency highlight this risk.

Infectious complications, such as herpes zoster, are known and essential considerations.

Suraj Rajasimhan

suraj.rajasimhan@nih.gov

1 Pharmacy Department, National Institutes of Health Clinical Center, 10 Center Drive, Bldg. 10, Room 1C240, Bethesda, MD 20892, USA

2 Office of the Clinical Director, Intramural Research Program, National Institute of Arthritis and Musculoskeletal and Skin Diseases, National Institutes of Health, Bethesda, MD, USA

\section{Introduction}

The Janus kinase (JAK)-signal transducer and activator of transcription (STAT) pathway is a membrane-to-nucleus signaling cascade that effects activation of gene transcription. Many cytokines, including interleukins, interferons, and colony-stimulating factors, signal through this pathway [1]. Selective JAK inhibitors ("jakinibs") have demonstrated effectiveness in a variety of autoimmune diseases [2] such as rheumatoid arthritis (RA), inflammatory bowel disease, and dermatological diseases. In addition, at least one jakinib (ruxolitinib) is approved for the treatment of polycythemia and myelofibrosis [1]. Because interferon signaling occurs through the JAK-STAT pathway, interest in the use of jakinibs for clinical situations characterized by an interferon signature is growing. For these reasons and more, clinical familiarity with the side effects of this category of therapeutic agents is of particular concern to geriatricians. Among the range of adverse effects associated with JAK inhibitors, reactivation of viral infections such as herpes zoster (HZ) must be considered before initiating therapy. Thromboembolic risks, for which new advice has been issued, must also be considered in geriatrics. In this article, we aim to summarize clinical data encompassing the risks of $\mathrm{HZ}$ reactivation and thromboembolism in older patients with RA. 


\section{Overview of the Adverse Effects of Jakinibs}

The broad nature of cytokine and other factor inhibition that is associated with the use of jakinibs is likely the cause of protean adverse events. An increased risk of infections associated with jakinib use may relate to inhibition of the signaling of many cytokines important for natural killer (NK), T-, and B-cell function [1]. For this reason, particular attention to the potential for varicella zoster virus (VZV) reactivation is required. This concern is particularly heightened in patients with autoimmune disease, who may be comanaged with other immunosuppressants (including glucocorticoids) [3]. Immune senescence, alcohol use, and comorbid medical conditions further compound this issue. Other effects associated with selective JAK inhibitors may include adverse impacts on lipid profiles, increased serum creatinine (reduced glomerular filtration rate), transaminitis, and gastrointestinal perforation [3].

In 2017, the European Medicines Agency (EMA) revised the summary of product characteristics for baricitinib to include deep venous thrombosis (DVT) and pulmonary embolism (PE). The agency cautioned against the use of these drugs in patients with risk factors for a DVT or PE, such as older individuals, patients with obesity or a medical history of DVT/PE, and those undergoing surgery and immobilization [4]. In 2019, the US FDA issued a "black box" warning that thrombosis, including PE, DVT, and arterial thrombosis, had occurred in patients treated with jakinibs [5]. This was based on interim results from the ongoing postmarketing clinical trial evaluating tofacitinib 5 and $10 \mathrm{mg}$ twice-daily (BID) in patients with RA. The increased risk was associated with the $10 \mathrm{mg}$ BID dosing when compared with a tumor necrosis factor (TNF) blocker.

\section{Mechanistic Considerations}

\subsection{Cellular and Cytokine Effects}

As alluded to above, abnormalities in lymphocytes may account for an increase in the risk of certain infections associated with the use of jakinibs. This is not surprising given the antiviral function of interferons (IFNs), one of the cytokines known to be inhibited by these agents. However, in addition to NK cells, jakinibs may also be associated with reduced numbers of neutrophils and platelets [6]. It is assumed that signaling related to erythropoietin, thrombopoietin, interleukin (IL)-6, and IL-11 plays a role in these observations [6].
The putative increased risk for thrombosis is an area of significant research effort, but inroads are only preliminary. For example, the adverse impact on platelets appears to relate to inhibition at the level of progenitor stem cells as opposed to the megakaryocyte [6]. Similarly, the increase in low-density lipoprotein associated with jakinibs is poorly understood. While this increase may raise clinical concern, the observation must be contrasted with a reported concomitant increase in high-density lipoprotein and a more efficient cholesterol transport mechanism [6].

Finally, cytokine dysregulation may impact the development of malignancy. Either indirectly, by allowing unchecked viral exposure [e.g., Epstein-Barr virus (EBV)], or directly, by inhibiting endogenous immune surveillance against cancer (e.g., blunting the type I IFN antitumor effect), many such immunosuppressants remain suspect in this regard [7].

\subsection{Cytochrome P450 System}

Selective JAK inhibitors may involve cytochrome P450 (CYP)-3A4-mediated drug-drug interactions with the potential to adversely impact commonly used medications such as statins in older patients [1]. Tofacitinib is primarily metabolized in the liver through CYP3A4 so requires dose adjustments when coadministered with CYP3A4 inhibitors [8]. On the other hand, the toxicity of ruxolitinib may increase if coadministered with potent CYP3A4 inhibitors [9]. In contrast, baricitinib is a first-generation jakinib that is metabolized not via the CYP system [2] but via the kidney. Similarly, pharmacokinetic studies have not uncovered clinically meaningful effects on upadacitinib by moderate CYP3A4 inhibitors [10].

\subsection{Pharmacokinetic Considerations}

The pharmacology and pharmacokinetics of any given drug may vary with patient age, posing a unique challenge for prescribers [11]. According to the US Department of Health and Human Services, a greater proportion of patients will have RA at an advanced age. Curtis et al. [12] found that older patients receiving tofacitinib experienced higher rates of serious adverse effects and greater discontinuation of therapy because of an adverse drug event than did a younger population. Complicating the picture, comorbid conditions such as cardiovascular disease and diabetes are more prevalent in those aged $>65$ years. For example, tofacitinib is primarily metabolized in the liver and excreted via the kidneys [13]. A pharmacokinetic study conducted in healthy volunteers showed that the cumulative excretion of tofacitinib in the urine was $80.1 \%$ [14]. Older patients with reduced glomerular filtration rates require close monitoring because of potential drug accumulation. Kidney clearance 
of tofacitinib reduced with increased renal impairment [13]. The manufacturers of tofacitinib, baricitinib, fedratinib, and ruxolitinib all recommend dose adjustments in renal impairment. No specific dosing recommendations are available for upadacitinib. However, compared with subjects with normal renal function, subjects with mild or moderate renal impairment showed a 16 and 32\% higher area under the concentration-time curve, respectively, with this agent [15]. Similarly, renal filtration and active secretion are the principal clearance mechanisms for baricitinib.

\section{Special Geriatric Considerations}

\subsection{Janus Kinase (JAK) Inhibitors and the Risk of Herpes Zoster}

$\mathrm{HZ}$ results from the reactivation of latent $\mathrm{VZV}$, the risk of which increases with age $[16,17]$. Immune-mediated diseases such as RA and systemic lupus erythematosus (SLE), along with immunosuppressive medications, further increase the risk of HZ [18, 19]. The proposed mechanism involves the immune response signaling of HZ via type I and type II IFNs utilizing JAK-STAT pathways [20]. Hence, medications targeting this pathway are predicted to increase the risk of HZ. Tofacitinib, a JAK3 and JAK1 inhibitor with some affinity for JAK2 is known to increase the risk of $\mathrm{HZ}$ in a dose-dependent fashion [21, 22]. Concomitant use of glucocorticoids or methotrexate may further increase this risk. In the tofacitinib RA development program, Winthrop et al. [23] determined incidence rates (IRs) of $\mathrm{HZ}$ infection to be 4.4 per 100 patient-years. In the same study, the IR in patients aged $\geq 60$ years receiving prednisone was 6.3 per 100 patient-years [23]. A more recent meta-analysis comprising over 66,000 patients treated with JAK inhibitors found an increased risk of HZ infection [24]. The IR was 2.11 per 100 patient-years compared with 1.23 per 100 patient-years in the comparator group [24]. The majority of patients in this analysis had a diagnosis of RA and received tofacitinib. A higher proportion of these patients were older and female [24].

Genovese et al. [25] reported a 7-year safety analysis of baricitinib that included 3770 patients with moderate to severe RA. The incidence of $\mathrm{HZ}$ was significantly higher with baricitinib $4 \mathrm{mg}$ than with placebo (3.8 vs. $0.9 \%)$ and numerically higher with baricitinib $2 \mathrm{mg}(3.1 \%)$ [25]. In a pooled analysis of RA clinical trials of baricitinib, the HZ IRs were similar to those for tofacitinib [26]. Patients treated with baricitinib $4 \mathrm{mg}$ daily over a 2 -year median time period showed an HZ IR of 4.3 per 100 patient-years within the first 24 weeks [26]. Newer, more selective JAK inhibitors may also carry an increased risk for HZ [27]. Despite concern for a class effect with regard to reactivation of VZV, clinically significant effects on other viruses such as cytomegalovirus or EBV have not been seen [28].

Vaccinating patients before initiating JAK inhibitors may reduce the risk of HZ. Unfortunately, according to the Morbidity and Mortality Weekly Report in 2015, only 34.2\% of adults aged $\geq 65$ years in the USA were vaccinated for HZ [29]. Routine assessment of vaccination status and active strategies to vaccinate patients before initiating JAK inhibitor therapy is prudent.

\subsection{JAK Thrombosis}

Patients with RA/SLE are at increased risk for major cardiovascular adverse events, including PE and venous thromboembolism (VTE), compared with the general population $[30,31]$. Recently, the FDA released a black box warning for increased thromboembolic risk associated with JAK inhibitors. The main driver for this decision was the results of a postmarketing study that compared tofacitinib $5 \mathrm{mg}$ BID and $10 \mathrm{mg}$ BID in the treatment of RA. The study showed older patients with one or more cardiovascular risk factors who received the higher dose developed more DVT or PE. Furthermore, the FDA broadened this warning for other approved JAK inhibitors such as upadacitinib and baricitinib. This warning highlights individuals aged $>50$ years. In the same light, the EMA safety committee stated that patients aged $>65$ years should be treated with tofacitinib only when there is no alternative treatment.

According to the FDA Adverse Events Reporting System (FAERS) public dashboard, 436 cases of DVT and PE have been reported since 2010 among all JAK inhibitors approved for RA. Of these, 140 subjects were aged $>65$ years and were mostly female (Fig. 1). This report was generated by filtering cases of DVT and PE reported under baricitinib, tofacitinib, and upadacitinib treatment and is current as of 31 December 2019 [32]. The majority of these cases (385) related to tofacitinib, and this may be a consequence of historical primacy, as it has been on the market since 2012 . While this is a significant number of events, this list may contain many duplicates, and a comprehensive review of each report is necessary to establish causation. FAERS data reviewed by Verden et al. [33] in 2017 indicated an elevated reporting of thromboembolic adverse events for both tofacitinib and ruxolitinib. However, in this review, the rates of reporting of DVT and PE were not elevated.

Here, it is important to emphasize that, a priori, patients with RA have an up to three times greater risk of thromboembolism than do healthy controls. Moreover, patients with RA have an increased risk of DVT, independent of treatment. The IRs of VTE, including DVT and PE, among patients with RA range from 0.3 to 0.8 per 100 patient-years [34-36]. In one study, VTE events were 2.4 times more frequent in patients with RA than in those without RA [37]. In 


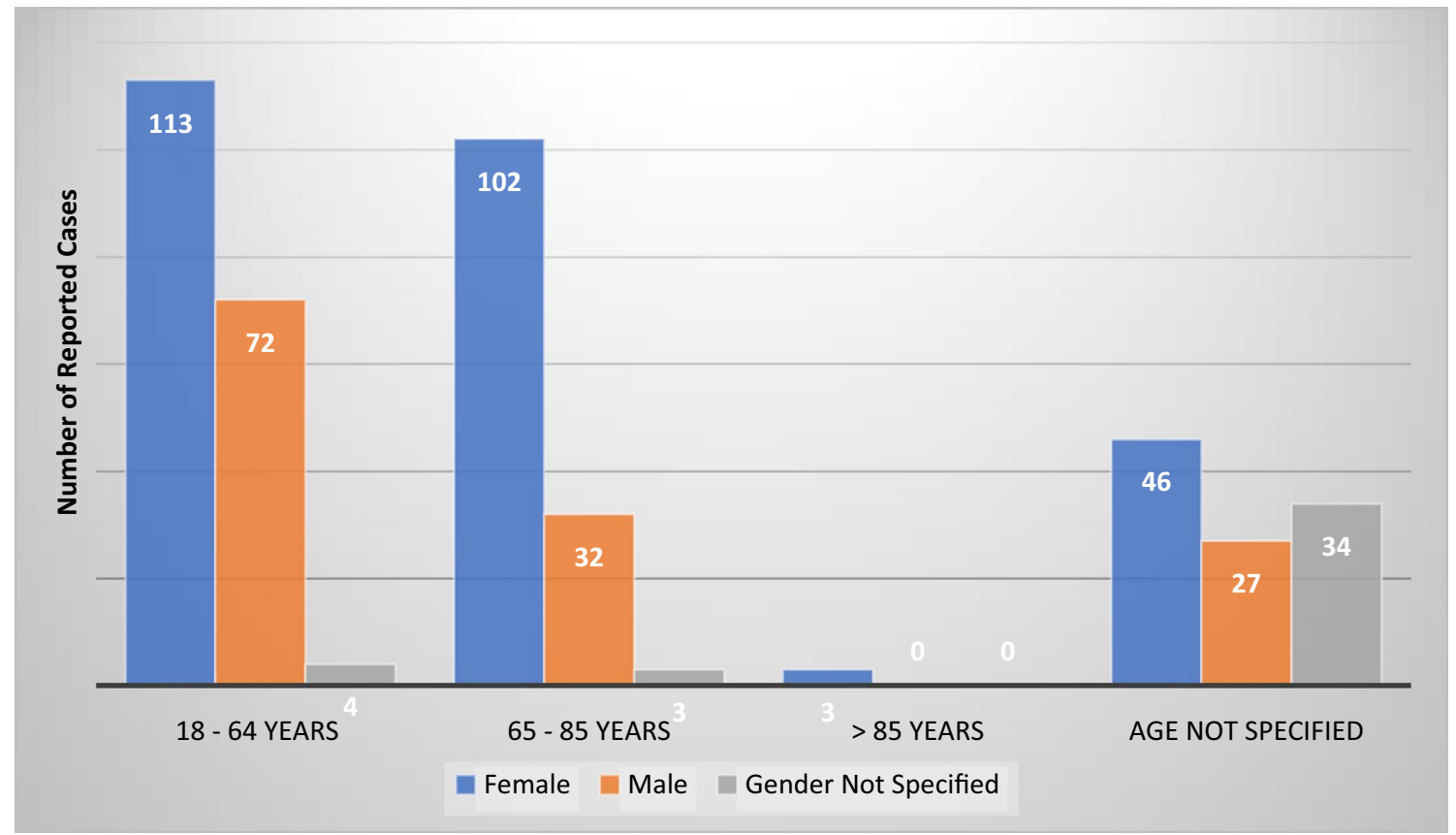

Fig. 1 Baricitinib, tofacitinib and upadacitinib US FDA Adverse Event Reporting System public dashboard data; reported cases of deep vein thrombosis and pulmonary embolism 2010-2019

this study, the risk of VTE remained increased in patients with RA after adjusting for known thromboembolic risk factors (hazard ratio [HR] 1.4; 95\% confidence interval [CI] 1.1-1.7). A recent meta-analysis of 25 clinical trials indicated a trend toward higher rates of VTE with JAK inhibitors than with placebo (odds ratio 1.16; 95\% CI 0.48-2.8) [38]. This finding is reminiscent of the confounding bias in play between RA and lymphoma when considering exposure to TNF blockers. The risk of lymphoma is increased in RA and especially so in patients most severely affected by RA, namely, those who may have the greatest need for TNF blockers. In addition, the background risk of VTE is also increased in other diseases for which jakinibs are approved, such as ulcerative colitis [39] and psoriatic arthritis [36].

\subsection{Tofacitinib and VTE Risk}

In a postmarketing safety trial, patients with RA aged $>50$ years who had one or more cardiovascular risk factor were randomized to tofacitinib $5 \mathrm{mg}$ or $10 \mathrm{mg}$ BID or TNF blockers. The preliminary analysis revealed a fivetimes increased risk of PE in patients who received tofacitinib $10 \mathrm{mg}$ BID when compared with TNF blockers. At the interim analysis, $\mathrm{PE}$ had developed in 19 patients using tofacitinib $20 \mathrm{mg}$ daily and in three patients using TNF blockers. The risk of mortality was also high with tofacitinib $10 \mathrm{mg}$ BID (45 patients died) compared with TNF blockers (25 patients died), covering nearly 3900 patient-years. No data are available for the tofacitinib $5 \mathrm{mg}$ BID group. All patients receiving high-dose tofacitinib were switched to $5 \mathrm{mg}$ BID after this preliminary analysis [5, 40].

Before this report, some studies analyzed available clinical data for the risk of thrombosis in patients with RA using tofacitinib [41]. Desai et al. [41] evaluated two different databases (Truven and Medicare) to investigate the risk of VTE in patients with RA who previously received tofacitinib. The definition of VTE relied upon inpatient claims for DVT or PE. The control group consisted of patients with RA using TNF blockers in the same databases. The mean age of patients in the Medicare database was higher than those in Truven. Total VTE frequency was numerically higher in the tofacitinib group, but this difference was not statistically significant. Consistent with the older age of the cohort, the frequency of VTE was higher in Medicare patients with RA. When the databases were combined, the pooled HR (1.33; 95\% CI 0.78-2.24) did not reveal significantly increased VTE risk in the tofacitinib group. Limitations of this study included the small number of VTE and the lack of a dosespecific analysis (Table 1). In addition, detail concerning the age of patients with VTE in the study was lacking.

Another study analyzed the IRs of VTE in phase II and III randomized controlled tofacitinib studies in RA, psoriasis, psoriatic arthritis, and ulcerative colitis and compared their IRs with Corrona Registry data. The study did not show any increased risk of VTE in patients who used tofacitinib in any disease, including RA. The study also included an analysis of tofacitinib $10 \mathrm{mg}$ BID where the risk of VTE was not different in this group when compared with the tofacitinib $5 \mathrm{mg}$ 
Table 1 Mean ages and venous thromboembolism incidence rates of patients with rheumatoid arthritis initiating treatment with tofacitinib or TNF blockers

\begin{tabular}{lllll}
\hline Age and IR & Truven (tofacitinib) & Truven (TNF blocker) & Medicare (tofacitinib) & Medicare (TNF blocker) \\
\hline$n$ & 1910 & 32,164 & 995 & 16,091 \\
Mean age & $54 \pm 11.6$ & $50 \pm 12.7$ & $71 \pm 5.7$ & $71 \pm 5.9$ \\
IR per 100 PY & $0.60(0.26-1.19)$ & $0.34(0.27-0.41)$ & $1.12(0.45-2.31)$ & $0.92(0.76-1.11)$ \\
\hline
\end{tabular}

Data are presented as mean \pm standard deviation or IR (95\% confidence interval) unless otherwise indicated

$C I$ confidence interval, $I R$ incidence rate, $P Y$ person-year, $T N F$ tumor necrosis factor

Table 2 Incidence rates for deep vein thrombosis and pulmonary embolism in tofacitinib clinical development studies

\begin{tabular}{|c|c|c|c|c|c|c|c|}
\hline \multirow[t]{2}{*}{ Adverse event } & \multicolumn{3}{|c|}{ Placebo-controlled cohort } & \multicolumn{4}{|c|}{ Dose-comparison cohort } \\
\hline & $\begin{array}{l}\text { Tofacitinib } \\
5 \text { mg BID }\end{array}$ & $\begin{array}{l}\text { Tofacitinib } \\
10 \mathrm{mg} \mathrm{BID}\end{array}$ & Placebo & $\begin{array}{l}\text { Tofacitinib } \\
5 \text { mg BID }\end{array}$ & $\begin{array}{l}\text { Tofacitinib } \\
10 \mathrm{mg} \text { BID }\end{array}$ & $\begin{array}{l}\text { Adalimumab } \\
40 \mathrm{mg} \mathrm{SC} \mathrm{q} 2 \mathrm{w}\end{array}$ & $\begin{array}{l}\text { MTX } \\
20 \mathrm{mg} \mathrm{qw}\end{array}$ \\
\hline DVT & $\begin{array}{l}0 / 1849 \\
0(0-0.9)\end{array}$ & $\begin{array}{l}0 / 2024 \\
0(0-0.8)\end{array}$ & $\begin{array}{l}1 / 1079 \\
0.4(0-2.4)\end{array}$ & $\begin{array}{l}1 / 1849 \\
0.1(0-0.3)\end{array}$ & $\begin{array}{l}1 / 2024 \\
0.1(0-0.3)\end{array}$ & $\begin{array}{l}0 / 257 \\
0(0-0.9)\end{array}$ & $2 / 220.7(0.1-2.5)$ \\
\hline PE & $\begin{array}{l}0 / 1849 \\
0(0-0.9)\end{array}$ & $\begin{array}{l}0 / 2024 \\
0(0-0.8)\end{array}$ & $\begin{array}{l}1 / 1079 \\
0.4(0-2.4)\end{array}$ & $\begin{array}{l}2 / 1849 \\
0.1(0-0.4)\end{array}$ & $\begin{array}{l}3 / 2024 \\
0.2(0-0.4)\end{array}$ & $\begin{array}{l}0 / 257 \\
0(0-1.9)\end{array}$ & $\begin{array}{l}0 / 223 \\
0(0-1.3)\end{array}$ \\
\hline
\end{tabular}

The data include 5368 patients with rheumatoid arthritis and 4440 person-years. Data are presented as n/N and incidence rate $(95 \%$ confidence interval)

$B I D$ twice daily, $D V T$ deep vein thrombosis, $I R$ incidence rates, $M T X$ methotrexate, $P E$ pulmonary embolism, $q 2 w$ every 2 weeks, $q w$ every week, $S C$ subcutaneous

BID group [42]. The frequencies of DVT and PE in patients with RA are seen in Table 2.

\subsection{Baricitinib and Venous Thromboembolism (VTE) Risk}

Taylor et al. [43] assessed the frequency of cardiovascular and VTE events in clinical studies of baricitinib. The authors analyzed nine RA studies, six of which included placebo comparison for up to 24 weeks. In addition, four studies randomly compared baricitinib 2 and $4 \mathrm{mg}$. VTE was observed in six of 997 patients treated with baricitinib $4 \mathrm{mg}$ and none of the 1070 patients in the placebo group. All patients with RA who developed VTE had other risk factors. Five of six patients with VTE were aged $>50$ years (range 53-66). Also, all older VTE patients had a high body mass index $\left(>35 \mathrm{~kg} / \mathrm{m}^{2}\right)$. Further analysis of extension data found that the IRs of VTE were similar with both 2 and $4 \mathrm{mg}$ doses (respectively, 0.5 and 0.6 per 100 patient-years). These IRs are similar to the VTE IR of general patients with RA (0.3-0.8). The study compared the clinical features of 42 patients with and 3450 patients without VTE in all baricitinib study periods. Multivariate Cox regression analysis revealed that mean age and body mass index, concomitant cyclooxygenase-2 inhibitor usage, and history of previous VTE were independent risk factors for VTE. Data from all baricitinib rheumatoid arthritis (All BARI RA) studies also showed an increased tendency of VTE risk with older age [26]. In addition, the VTE IRs were lower in the All BARI RA group than in other databases in all age groups (Table 3).

In summary, there was no statistically increased risk of VTE in baricitinib studies. Also, the IR of VTE did not increase over time despite continuing baricitinib usage. Nevertheless, the FDA only approved the baricitinib $2 \mathrm{mg}$ dose regimen for RA treatment based on the numerically increased VTE incidence in the higher-dose baricitinib group [44].

\subsection{Upadacitinib and VTE Risk}

According to an FDA review [45], the IR of VTE was $0.8 / 100$ person-years in patients treated with upadacitinib $15 \mathrm{mg}$ and $0.5 / 100$ person-years in the higher-dose upadacitinib $30 \mathrm{mg}$ group during a short-term controlled period of phase III studies. IRs were 3.5 and $0.4 / 100$ person-years in adalimumab and placebo groups in this review. Over a long-term extension period for these studies, the IRs of VTE were 0.6 and $0.3 / 100$ person-years in the upadacitinib 15 and $30 \mathrm{mg}$ groups, respectively. In five phase III clinical trials, 906 patients $(20.7 \%)$ were aged $>65$ years, and the effectiveness of upadacitinib was similar in all age groups. However, the frequency of overall side effects was higher in the older patients with RA. In an integrated analysis of upadacitinib phase III clinical trials, major adverse cardiovascular 
Table 3 Incidence rates of venous thromboembolism in all baricitinib rheumatoid arthritis studies (ALL BARI RA) and of rheumatoid arthritis patient databases (IMEDS and Truven) who did not use baricitinib

\begin{tabular}{llll}
\hline Age groups & ALL BARI RA $(n=3492)$ & IMEDS $(n=69,095)$ & Truven $(n=205,785)$ \\
\hline $18-49$ & $0.14(0.04-0.35)$ & $0.49(0.37-0.63)$ & 0.50 \\
$50-59$ & $0.57(0.32-0.95)$ & $0.90(0.75-1.07)$ & 0.77 \\
$60-64$ & $0.77(0.35-1.47)$ & $1.16(0.93-1.43)$ & 1.05 \\
$\geq 65$ & $1.09(0.59-1.83)$ & $2.13(1.94-2.33)$ & 2.16 \\
All ages & $0.53(0.38-0.71)$ & $1.34(1.24-1.44)$ & $1.05(1.01-1.09)$ \\
\hline
\end{tabular}

Data are presented as number per 100 person-years (95\% CI). The Truven data do not include 95\% CIs by age groups [44]

CI confidence interval, IMEDS Innovation in Medical Evidence Development and Surveillance, Truven Truven health marketscan commercial claims and encounters databases

events and VTE rates were comparable to those with placebo, methotrexate, and adalimumab [46]. All patients with a VTE event had one or more cardiovascular or VTE risk factor at baseline. Although lipid levels were increased in the upadacitinib group, no association between this and cardiovascular events was established. Consequently, the FDA recently approved upadacitinib for the treatment of RA but released a black box warning for increased risk of thrombosis consistent with all JAK inhibitor product labeling.

\section{Discussion}

The safety of JAK inhibitors in older patients remains to be clarified. The risk of infections, specifically $\mathrm{HZ}$ reactivation, is noteworthy with this class of drugs. Infections, specifically $\mathrm{HZ}$, are a common occurrence and may be considered a class effect. Hepatic metabolism and renal elimination are other significant pharmacokinetic and pharmacodynamic considerations in older patients. Drug accumulation due to reduced renal or hepatic function can increase overall adverse events.

Recent postmarketing investigations showed an increased risk of VTE and mortality in older patients with RA using high-dose tofacitinib and with one or more cardiovascular risk factors. The data for thrombosis risk with baricitinib $4 \mathrm{mg}$ also supports this suspicion. Overall, it appears that the older-age RA population is at highest risk. However, the complete mechanism and magnitude of thromboembolic disease is yet to be clarified.

An important question to be resolved is whether or not the increased risk of VTE in patients with RA is a medication class effect, limited solely to high-dose tofacitinib use or the underlying disease that modifies the risk. While the FDA warning covers all of the approved JAK inhibitors, analysis of randomized studies has so far shown only numerically, not statistically, increased VTE risk for all agents except tofacitinib and baricitinib. That being said, some VTE cases were detected in upadacitinib studies [45].
Speculation for a mechanistic explanation for increased risk of thrombosis has focused on an elevation of platelet count observed in the early phase of treatment. In baricitinib studies, this elevation was mainly seen in the $4 \mathrm{mg}$ group. However, the platelet count returned to the normal during longer-term follow-up.

Studies evaluating the relationship between JAK inhibitors and VTE in patients with RA have some limitations. First, the duration of the placebo-controlled phase of a study may be too short. Second, controlling for confounding factors likely requires enrollment and observation of larger cohorts. Third, insufficient time on the market since FDA approval limits postmarketing safety data for many JAK inhibitors (e.g., baricitinib, upadacitinib), especially as pertains to the special target population of older patients with RA. Indeed, the exclusion of older patients from randomized RA studies limits the generalizability of results.

\section{Concluding Comments}

A nuanced understanding of the use and side effects of jakinibs is of critical importance to those treating older adults. More studies are needed to investigate the relationship between thrombosis and JAK inhibitors. For example, the effect of JAK inhibitors on endothelial injury or on the coagulation system are avenues of likely fruitful further study.

With an anticipated forthcoming explosion of so-called next-generation jakinibs, the clinical challenge to those providing healthcare for the older population is expected to increase [47]. At the same time, the improved selectivity of these agents may shed light on a refined appreciation for safe use of these agents [6]. Finally, as new uses for this class of drugs are uncovered, such as in the fight against osteoporosis, it is increasingly likely that geriatricians will find themselves at the forefront of prescribing and monitoring their use [48]. 


\section{Compliance with Ethical Standards}

Conflict of interest The NIH, but not the authors of this review, reports the following disclosure statement: The National Institutes of Health holds patents on targeting JAKs. NIAMS and Pfizer have a Collaborative Research and Development Award (CRADA). However, the authors have no direct involvement in these patents or awards. Suraj Rajasimhan, Omer Pamuk, and James D. Katz have no conflicts of interest that are directly relevant to the content of this article.

Funding This research was supported by the Intramural Research Program of the National Institutes of Health, National Institute of Arthritis and Musculoskeletal and Skin Diseases.

Open Access This article is licensed under a Creative Commons Attribution-NonCommercial 4.0 International License, which permits any non-commercial use, sharing, adaptation, distribution and reproduction in any medium or format, as long as you give appropriate credit to the original author(s) and the source, provide a link to the Creative Commons licence, and indicate if changes were made. The images or other third party material in this article are included in the article's Creative Commons licence, unless indicated otherwise in a credit line to the material. If material is not included in the article's Creative Commons licence and your intended use is not permitted by statutory regulation or exceeds the permitted use, you will need to obtain permission directly from the copyright holder. To view a copy of this licence, visit http://creativecommons.org/licenses/by-nc/4.0/.

\section{References}

1. Gadina M, Johnson C, Schwartz D, Bonelli M, Hasni S, Kanno Y, Changelian P, Laurence A, O'Shea JJ. Translational and clinical advances in JAK-STAT biology: the present and future of jakinibs. J Leukoc Biol. 2018;104(3):499-514.

2. Schwartz DM, Kanno Y, Villarino A, Ward M, Gadina M, O'Shea JJ. JAK inhibition as a therapeutic strategy for immune and inflammatory diseases. Nat Rev Drug Discov. 2017;17(1):78.

3. Betancourt BY, Biehl A, Katz JD, Subedi A. Pharmacotherapy pearls in rheumatology for the care of older adult patients: focus on oral disease-modifying antirheumatic drugs and the newest small molecule inhibitors. Rheum Dis Clin N Am. 2018;44(3):371-91.

4. European Medicines Agency (2017). Olumiant: summary of product characteristics. https://www.ema.europa.eu/en/documents/ product-information/olumiant-epar-product-information_en.pdf. Accessed Feb 7, 2020.

5. FDA Drug Safety Communication: FDA approves boxed warning about increased risk of blood clots and death with higher dose of arthritis and ulcerative colitis medicine tofacitinib (Xeljanz, Xeljanz XR). July 26, 2019. https://www.fda.gov/safety/medic al-product-safety-information/xeljanz-xeljanz-xr-tofacitinib-drugsafety-communication-due-increased-risk-blood-clots-and-death. Accessed Feb 7, 2020.

6. Gadina M, Le MT, Schwartz DM, Silvennoinen O, Nakayamada S, Yamaoka K, O'Shea JJ. Janus kinases to jakinibs: from basic insights to clinical practice. Rheumatology (Oxford). 2019;58(Suppl 1):i4-i16.

7. Cui J, Chen Y, Wang HY, Wang RF. Mechanisms and pathways of innate immune activation and regulation in health and cancer. Hum Vaccin Immunother. 2014;10(11):3270-85.
8. Gupta P, Chow V, Wang R, Kaplan I, Chan G, Alvey C, et al. Evaluation of the effect of fluconazole and ketoconazole on the pharmacokinetics of tofacitinib in healthy adult subjects. Clin Pharmacol Drug Dev. 2014;3(1):72-7.

9. Ajayi S, Becker H, Reinhardt H, Engelhardt M, Zeiser R, von Bubnoff N, Wäsch R. Ruxolitinib. Recent results. Cancer Res. 2018;212:119-32.

10. Klünder B, Mittapalli RK, Mohamed MF, Friedel A, Noertersheuser P, Othman AA. Population pharmacokinetics of upadacitinib using the immediate-release and extended-release formulations in healthy subjects and subjects with rheumatoid arthritis: analyses of phase I-III clinical trials. Clin Pharmacokinet. 2019;58(8):1045-58.

11. Cho S, Lau SW, Tandon V, Kumi K, Pfuma E, Abernethy DR. Geriatric drug evaluation: where are we now and where should we be in the future? Arch Intern Med. 2011;171(10):937-40.

12. Curtis JR, Schulze-Koops H, Takiya L, Mebus CA, Terry KK, Biswas P, Jones TV. Efficacy and safety of tofacitinib in older and younger patients with rheumatoid arthritis. Clin Exp Rheumatol. 2017;35(3):390-400.

13. Krishnaswami S, Chow V, Boy M, Wang C, Chan G. Pharmacokinetics of tofacitinib, a janus kinase inhibitor, in patients with impaired renal function and end-stage renal disease. J Clin Pharmacol. 2014;54(1):46-52.

14. Dowty ME, Lin J, Ryder TF, Wang W, Walker GS, Vaz A, Chan GL, Krishnaswami S, Prakash C. The pharmacokinetics, metabolism, and clearance mechanisms of tofacitinib, a janus kinase inhibitor, in humans. Drug Metab Dispos. 2014;42(4):759-73.

15. Klünder B, Mohamed MF, Othman AA. Population pharmacokinetics of upadacitinib in healthy subjects and subjects with rheumatoid arthritis: analyses of phase I and II clinical trials. Clin Pharmacokinet. 2018;57(8):977-88.

16. Schmader K. Herpes zoster in older adults. Clin Infect Dis. 2001;32(10):1481-6.

17. Hope-Simpson RE. The nature of herpes zoster: a long-term study and a new hypothesis. Proc R Soc Med. 1965;58:9-20.

18. Thomas SL, Hall AJ. What does epidemiology tell us about risk factors for herpes zoster? Lancet Infect Dis. 2004;4(1):26-33.

19. Salam N, Rane S, Das R, Faulkner M, Gund R, Kandpal U, et al. T cell ageing: effects of age on development, survival and function. Indian J Med Res. 2013;138(5):595-608.

20. Ghoreschi K, Laurence A, O'Shea JJ. Janus kinases in immune cell signaling. Immunol Rev. 2009;228(1):273-87.

21. Winthrop KL, Curtis JR, Lindsey S, Tanaka Y, Yamaoka K, Valdez $\mathrm{H}$, et al. Herpes zoster and tofacitinib: clinical outcomes and the risk of concomitant therapy. Arthritis Rheumatol. 2017;69(10):1960-8.

22. Marra F, Lo E, Kalashnikov V, Richardson K. Risk of herpes zoster in individuals on biologics, disease-modifying antirheumatic drugs, and/or corticosteroids for autoimmune diseases: a systematic review and meta-analysis. Open Forum Infect Dis. 2016;3(4):ofw205.

23. Winthrop KL, Yamanaka H, Valdez H, Mortensen E, Chew R, Krishnaswami S, et al. Herpes zoster and tofacitinib therapy in patients with rheumatoid arthritis. Arthritis Rheumatol. 2014;66(10):2675-84.

24. Olivera PA, Lasa JS, Bonovas S, Danese S, Peyrin-Biroulet L. Safety of Janus kinase inhibitors in patients with inflammatory bowel diseases or other immune-mediated diseases: a systematic review and meta-analysis. Gastroenterology. 2020;158(6):15541573.e12. https://doi.org/10.1053/j.gastro.2020.01.001.

25. Genovese M, Smolen J, Takeuchi T, Burmester G, Brinker D, Rooney T, Zhong J, Mo D, Saifan C, Cardoso A, Issa M, Wu W, Winthrop K. Safety profile of baricitinib for the treatment of rheumatoid arthritis up to 7 years: an updated integrated safety analysis [abstract]. Arthritis Rheumatol. 2019;71(suppl 10). https 
://acrabstracts.org/abstract/safety-profile-of-baricitinib-for-thetreatment-of-rheumatoid-arthritis-up-to-7-years-an-updated-integ rated-safety-analysis/. Accessed Feb 11, 2020.

26. Smolen JS, Genovese MC, Takeuchi T, Hyslop DL, Macias WL, Rooney T, et al. Safety profile of baricitinib in patients with active rheumatoid arthritis with over 2 years median time in treatment. J Rheumatol. 2019;46(1):7-18.

27. Moodley D, Yoshida H, Mostafavi S, Asinovski N, Ortiz-Lopez A, Symanowicz P, et al. Network pharmacology of JAK inhibitors. Proc Natl Acad Sci USA. 2016;113(35):9852-7.

28. Valenzuela F, Papp KA, Pariser D, Tyring SK, Wolk R, Buonanno $\mathrm{M}$, et al. Effects of tofacitinib on lymphocyte sub-populations, CMV and EBV viral load in patients with plaque psoriasis. BMC Dermatol. 2015;8(15):8.

29. Williams WW, Lu PJ, O'Halloran A, Kim DK, Grohskopf LA, Pilishvili T, et al. Surveillance of vaccination coverage among adult populations-United States, 2015. MMWR Surveill Summ. 2017;66(11):1-28.

30. Chung WS, Peng CL, Lin CL, Chang YJ, Chen YF, Chiang JY, Sung FC, Kao CH. Rheumatoid arthritis increases the risk of deep vein thrombosis and pulmonary thromboembolism: a nationwide cohort study. Ann Rheum Dis. 2014;73(10):1774-800.

31. Lee JJ, Pope JE. A meta-analysis of the risk of venous thromboembolism in inflammatory rheumatic diseases. Arthritis Res Ther. 2014;16(5):435.

32. FDA Adverse Events Reporting System (FAERS) Public Dashboard. https://fis.fda.gov/sense/app/d10be6bb-494e-4cd282e4-0135608ddc13/sheet/7a47a261-d58b-4203-a8aa-6d302 1737452/state/analysis. Accessed Feb 12, 2020.

33. Verden A, Dimbil M, Kyle R, Overstreet B, Hoffman KB. Analysis of spontaneous postmarket case reports submitted to the FDA regarding thromboembolic adverse events and JAK inhibitors. Drug Saf. 2018;41(4):357-61. https://doi.org/10.1007/s4026 4-017-0622-2.

34. Holmqvist ME, Neovius M, Eriksson J, Mantel Ä, Wållberg-Jonsson S, Jacobsson LT, Askling J. Risk of venous thromboembolism in patients with rheumatoid arthritis and association with disease duration and hospitalization. JAMA. 2012;308(13):1350-6.

35. Choi HK, Rho YH, Zhu Y, Cea-Soriano L, Aviña-Zubieta JA, Zhang Y. The risk of pulmonary embolism and deep vein thrombosis in rheumatoid arthritis: a UK population-based outpatient cohort study. Ann Rheum Dis. 2013;72(7):1182-7.

36. Ogdie A, Kay McGill N, Shin DB, Takeshita J, Jon Love T, Noe MH, Chiesa Fuxench ZC, Choi HK, Mehta NN, Gelfand JM. Risk of venous thromboembolism in patients with psoriatic arthritis, psoriasis and rheumatoid arthritis: a general population-based cohort study. Eur Heart J. 2018;39(39):3608-14.

37. Kim SC, Schneeweiss S, Liu J, Solomon DH. Risk of venous thromboembolism in patients with rheumatoid arthritis. Arthritis Care Res (Hoboken). 2013;65(10):1600-7.

38. Xie W, Huang Y, Xiao S, Sun X, Fan Y, Zhang Z. Impact of Janus kinase inhibitors on risk of cardiovascular events in patients with rheumatoid arthritis: systematic review and meta-analysis of randomised controlled trials. Ann Rheum Dis. 2019;78(8):1048-54.
39. McCurdy JD, Kuenzig ME, Smith G, Spruin S, Murthy SK, Carrier M, et al. Risk of venous thromboembolism after hospital discharge in patients with inflammatory bowel disease: a populationbased study. Inflamm Bowel Dis. 2020 (Epub ahead of print).

40. FDA-2: FDA drug safety communication: Safety trial finds risk of blood clots in the lungs and death with higher dose of tofacitinib (Xeljanz, Xeljanz XR) in rheumatoid arthritis patients; FDA to investigate. February 25, 2019. https://www.fda.gov/media/12048 5/download. Accessed Feb 7, 2020.

41. Desai RJ, Pawar A, Weinblatt ME, Kim SC. Comparative risk of venous thromboembolism in rheumatoid arthritis patients receiving tofacitinib versus those receiving tumor necrosis factor inhibitors: an observational cohort study. Arthritis Rheumatol. 2019;71(6):892-900.

42. Mease PJ, Kremer J, Cohen S, Curtis JR, Charles-Schoeman C, Loftus EV, Greenberg JD, Palmetto N, Kanik KS, Graham D, Wang C, Biswas P, Chan G, DeMasi R, Valdez H, Hendrikx T, Jones TV. Incidence of thromboembolic events in the tofacitinib rheumatoid arthritis, psoriasis, psoriatic arthritis and ulcerative colitis development programs [abstract]. Arthritis Rheumatol. 2017;69(suppl 10). https://acrabstracts.org/abstract/incidenceof-thromboembolic-events-in-the-tofacitinib-rheumatoid-arthr itis-psoriasis-psoriatic-arthritis-and-ulcerative-colitis-developmen t-programs/. Accessed Feb 7, 2020.

43. Taylor PC, Weinblatt ME, Burmester GR, Rooney TP, Witt $\mathrm{S}$, Walls $\mathrm{CD}$, et al. Cardiovascular safety during treatment with baricitinib in rheumatoid arthritis. Arthritis Rheumatol. 2019;71(7):1042-55.

44. FDA Center for Drug Evaluation and Research. Summary of Resubmission and DPARB/OND Recommendations. Baricitinib, NDA 207924. https://www.accessdata.fda.gov/drugsatfda_docs/ nda/2018/207924Orig1s000SumR.pdf Accessed Feb 7, 2020. FDA Center for Drug Evaluation and Research. Patient Labeling Review. https://www.accessdata.fda.gov/drugsatfda_docs/ nda/2018/207924Orig1s000OtherR.pdf. Accessed May 3, 2020.

45. FDA-Upadacitinib: Risk Assessment and Risk Mitigation Review - FDA https://www.accessdata.fda.gov/drugsatfda_docs/ nda/2019/211675Orig1s000RiskR.pdf Accessed February 7, 2020.

46. Choy E, McInnes I, Cush J, Aelion J, Zhang Y, Khan N, Liu J, Camp H, Meerwein S, Rigby W, Cohen A. MACE and VTE Across Multiple Upadacitinib Studies in Rheumatoid Arthritis: Integrated Analysis from the SELECT Phase 3 Clinical Program [abstract]. Arthritis Rheumatol. 2019; 71 (suppl 10). https://acrab stracts.org/abstract/mace-and-vte-across-multiple-upadacitinibstudies-in-rheumatoid-arthritis-integrated-analysis-from-the-selec t-phase-3-clinical-program/. Accessed February 7, 2020.

47. O'Shea JJ, Gadina M. Selective Janus kinase inhibitors come of age. Nat Rev Rheumatol. 2019;15(2):74-5.

48. Adam S, Simon N, Steffen U, et al. JAK inhibition increases bone mass in steady-state conditions and ameliorates pathological bone loss by stimulating osteoblast function. Sci Transl Med. 2020;12(530):aay4447. 